\title{
Dynamic Markov Chain Monte Carlo-Based Spectrum Sensing
}

\author{
Zheng Wang, Member, IEEE, Ling Liu, Member, IEEE and Kezhi Li, Member, IEEE
}

\begin{abstract}
In this letter, a random sampling strategy is proposed for the non-cooperative spectrum sensing to improve its performance and efficiency in cognitive radio (CR) networks. The proposed refined Metropolis-Hastings (RMH) algorithm generates the desired channel sequence for fine sensing by sampling from the approximated channel availability distributions in an Markov chain Monte Carlo (MCMC) way. The proposal distribution during the sampling is fully exploited and the convergence of the Markov chain is studied in detail, which theoretically demonstrate the superiorities of the proposed RMH sampling algorithm in both sensing performance and efficiency.
\end{abstract}

Keywords: Spectrum sensing, Markov chain Monte Carlo

\section{INTRODUCTION}

Cognitive radio (CR) has emerged as a promising technique for wireless communications to opportunistically access the available spectrum resources without affecting the primary user networks. As a key process for cognitive radio, spectrum sensing aims to identify the available channels through cooperative or non-cooperative approaches [1]-[4], where channel access and data transmission are subsequently performed based on these available channels. Specifically, with respect to non-cooperative approaches, a salient issue is that their sensing performance are heavily determined by the assumed parametric traffic model. Once the estimation of the parametric traffic model becomes inaccurate, the spectrum sensing performance would be severely deteriorated. However, with the development of the highly dynamic heterogeneous CR ad hoc networks, it tends to be quite challenging to obtain the accurate traffic model instantaneously, rendering the non-parametricbased approach a valuable alternative for the non-cooperative spectrum sensing [5].

In order to overcome the sensing dependence on the parametric traffic model, a non-parametric sensing approach was proposed in [6], which performs the statistical inference through randomly sampling over channel availability distributions. In particular, it formulates the problem of seeking the optimal available channel sequence for fine sensing as an optimization problem to maximize the probability of channel availabilities, where the required channel availability distribution is approximated by the latest results of fast sensing. Then, the choices in the desired channel sequence are sampled one by one, and the channels with large availabilities would more likely be returned, thus leading to a guaranteed sensing performance. However, since sampling from the channel availability distribution directly is difficult, the method of MCMC

Z. Wang is with the College of Electronic Information Engineering, Nanjing University of Aeronautics and Astronautics, 211106, China; L. Liu is with the College of Computer Science and Software Engineering, Shenzhen University, 518060, China; Kezhi Li is with the Institute of Health Informatics, University College London, WC1E 6BT, United Kingdom (e-mail: z.wang@ieee.org). was introduced to obtain the samples from the mixing of Markov chain, then the target channel sequence is generated by selecting valid candidates from these samples.

In this paper, the refined Metropolis-Hastings (RMH) is proposed to improve both the performance and efficiency of the non-parametric spectrum sensing. Typically, according to the flexibility of the proposal distribution in Metropolis-Hastings (MH) sampling, a refined proposal distribution is proposed, which results in a more accurate sampling approximation due to a faster Markov chain convergence rate. Meanwhile, the selection judgement of the channel sequence is carefully considered to improve the acceptance rate of the samples from the Markov mixing being accepted by the channel sequence, thus leading to a more efficient spectrum sensing.

\section{SySTEM MODEL}

Consider a spectrum containing $M$ non-overlapping channels, for each secondary user, a fast sensing over these $M$ channels is performed periodically by the way of energy detection. After that, a selected channel sequence $\mathcal{S}=\left[s_{1}, \ldots, s_{T}\right]$ is generated based on the results of fast sensing, and a fine sensing process over the spectrum is then carried out along $\mathcal{S}$ to ensure the following channel access and data transmission. Typically, the problem of identifying the optimal channel sequence $\widehat{\mathcal{S}}$ for fine sensing was formulated as to maximize the probability of channel availability

$$
\widehat{\mathcal{S}}=\underset{s_{1}, \ldots, s_{T}}{\arg \max } P\left(X_{s_{1}}=1, \ldots, X_{s_{T}}=1\right),
$$

where $X_{s_{i}} \in\{0,1\}$ is a binary availability indicator of channel $s_{i}$. In [6], a feasible way to solve this optimization problem is to sample each element of $\mathcal{S}$ from the distributions

$$
\bar{P}\left(s_{i}\right)=f\left(s_{i}\right) / K, \quad 1 \leq s_{i} \leq M,
$$

where $f\left(s_{i}\right)=\frac{1}{\varpi} \int_{0}^{\infty} \int_{\lambda}^{\infty} l\left(s_{i}\right) e^{-\frac{\omega}{\omega}} d_{s_{i}} d_{\omega}$ represents the channel availability discovered by fast sensing, and $K>0$ is a normalized constant. More specifically, $\omega$ and $\varpi$ are the instantaneous signal-to-interference-plus-noise ratio (SINR) and average SINR respectively, $\lambda$ is determined by the probability of false alarm, and $l\left(s_{i}\right)$ stands for the PDF of the test statistics of channel $s_{i}$ [7]

$$
l\left(s_{i}\right)= \begin{cases}\frac{1}{2^{k / 2} \Gamma(k / 2)} s_{i}^{(k / 2)-1} e^{-s_{i} / 2}, & H_{0} ; \\ \frac{1}{2} e^{-\left(s_{i} / 2+\mu\right)}\left(\frac{s_{i}}{2 \mu}\right)^{k / 4-0.5} I_{(k / 2)-1}\left(\sqrt{2 \mu s_{i}}\right), & H_{1},\end{cases}
$$

where $H_{0}$ and $H_{1}$ indicate the absence and presence of the primary network signals on channel $i, k$ is the degrees of freedom, $\Gamma$ is Gamma function, and $I$ is Bessel function.

However, direct sampling from (2) is difficult so that the Metropolis-Hastings (MH) sampling from MCMC was introduced, which generates $\mathcal{S}$ through a Markov chain $\mathcal{M}=$ 
$\left[M_{1}=m_{1}, M_{2}=m_{2}, \ldots\right]$ mixing towards the target distribution $\bar{P}\left(s_{i}\right)$ in an exponential convergence rate

$$
\left\|P^{t}(m, \cdot)-\bar{P}(\cdot)\right\|_{T V} \leq \varrho^{t} .
$$

Here, $t$ is the index of Markov move, $\|\cdot\|_{T V}$ denotes the total variation distance, $0<\varrho<1$ stands for the convergence rate, $M_{t}$ and $m_{t}$ represent the Markov state at move $t$ and its realization respectively. In general, $\mathrm{MH}$ sampling employs a proposal distribution $Q(\cdot)$ to activate an acceptance-rejection mechanism during the Markov move [8]. Specifically, given the incomplete channel sequence $\left[s_{1}, \ldots, s_{i-1}\right]$, the operations of MCMC sampling for identifying the channel choice $s_{i}$ during one Markov move can be described as follows:

Markov mixing:

1) Sample from the proposal distribution $Q\left(m_{t+1} \mid m_{t}\right)$ to obtain a candidate state $m^{*}$ for the Markov state $M_{t+1}$.

2) Calculate the acceptance ratio $\alpha\left(m^{*} \mid m_{t}\right)$

$$
\alpha\left(m^{*} \mid m_{t}\right)=\min \left\{1, \frac{\bar{P}\left(m^{*}\right) Q\left(m_{t} \mid m^{*}\right)}{\bar{P}\left(m_{t}\right) Q\left(m^{*} \mid m_{t}\right)}\right\} .
$$

3) With probability $\alpha\left(m^{*} \mid m_{t}\right)$ to accept $m^{*}$ by $M_{t+1}$, i.e. $M_{t+1}=m^{*}$, otherwise let $M_{t+1}=m_{t}$.

\section{Channel selection:}

4) If $m^{*}$ is accepted by $M_{t+1}$ and if $m^{*} \notin\left\{s_{1}, \ldots, s_{i-1}\right\}$, add $s_{i}=m^{*}$ into the channel sequence as $\mathcal{S}=\left[s_{1}, \ldots, s_{i}\right]$, otherwise try to obtain a valid $s_{i}$ at the next Markov move.

Consequently, the above process is performed subsequently until the target channel sequence $\mathcal{S}=\left[s_{1}, \ldots, s_{T}\right]$ for fine sensing is generated. Note that each channel choice $s_{i}$ in $\mathcal{S}$ should be different from each other.

\section{REFined MH SAMPLing AlgORIthm}

In essence, there are two loops in the above MCMC-based sensing process, namely, the Markov mixing and the channel selection. The inner loop maintains a valid Markov chain while the outer loop selects the eligible sample $M_{t+1}=m^{*}$ with $m^{*} \notin\left\{s_{1}, \ldots, s_{i-1}\right\}$ into the channel sequence $\mathcal{S}$. Undoubtedly, the sensing performance depends on the convergence performance of the Markov mixing as a faster convergence rate naturally accounts for a better sampling approximation of the target distribution $\bar{P}(\cdot)$. On the other hand, the sensing efficiency is partially determined by the selection judgement $m^{*} \notin\left\{s_{1}, \ldots, s_{i-1}\right\}$ at step (4). However, the possibility $m^{*} \notin\left\{s_{1}, \ldots, s_{i-1}\right\}$ decreases rapidly along with the increment of $i$ so that the sample from Markov chain would be rejected more and more frequently, resulting in a poor sensing efficiency as the inner loop for Markov mixing would be invoked intensively. Unfortunately, such a latent efficiency problem was not considered [6].

\section{A. Algorithm Description and Efficiency Improvement}

We now present the proposed refined MH (RMH) sampling algorithm, which attempts to incorporate the restriction $m^{*} \notin\left\{s_{1}, \ldots, s_{i-1}\right\}$ into the sampling at each Markov move to reduce the possibility $m^{*} \in\left\{s_{1}, \ldots, s_{i-1}\right\}$.

Specifically, observe that the sampling candidate $m^{*}$ for $M_{t+1}$ could be the same with the Markov states of the previous
Markov moves, i.e., $m^{*} \in\left\{m_{1}, \ldots, m_{t}\right\}$. Once it happens, $m^{*}$ must be rejected by the requirement $m^{*} \notin\left\{s_{1}, \ldots, s_{i-1}\right\}$, thus resulting in a sampling waste. To this end, it is strongly motivated to expand the sampling diversity of the underlying Markov chain. Interestingly, the proposal distribution $Q(\cdot)$ in MH sampling can be any fixed distribution from which one can conveniently draw samples [9]. For this reason, we try to fully take advantage of such a freedom and propose to update the proposal distribution as

$$
Q^{\prime}\left(m^{*} \mid m_{t}\right)=\frac{Q\left(m^{*} \mid m_{t}\right)}{1-Q\left(m^{*}=m_{t} \mid m_{t}\right)}
$$

to generate the sampling candidate $m^{*}$ for $M_{t+1}$. This means the Markov state $m_{t}$ at the previous sampling step is eliminated from the candidate state space in sampling $m^{*}$.

By doing this, we can simply guarantee that the candidate sample $m^{*}$ from the proposal distribution is different from the last Markov state $\mathcal{M}_{t}=m_{t}$, thereby establishing a more diverse Markov chain $\mathcal{M}=\left[M_{1}, M_{2}, \ldots\right]$. Thanks to the introduced diversity, the possibility $m^{*} \in\left\{s_{1}, \ldots, s_{i-1}\right\}$ would be reduced, and this corresponds to a higher acceptance rate in tackling with the requirement $m^{*} \notin\left\{s_{1}, \ldots, s_{i-1}\right\}$. On the other hand, once $s^{*}$ from $Q^{\prime}\left(m^{*} \mid m_{t}\right)$ is obtained, then the updated acceptance ratio $\alpha^{\prime}$ is calculated as follows

$$
\alpha^{\prime}\left(m^{*} \mid m_{t}\right)=\min \left\{1, \frac{\bar{P}\left(m^{*}\right) Q^{\prime}\left(m_{t} \mid m^{*}\right)}{\bar{P}\left(m_{t}\right) Q^{\prime}\left(m^{*} \mid m_{t}\right)}\right\},
$$

and the decision about whether to accept it into the sequence $\mathcal{S}$ as $s_{i}=m^{*}$ is performed thereafter. Therefore, a more efficient spectrum sensing is achieved due to a larger acceptance rate in the selection judgement. Typically, in the proposed RMH sampling, given the incomplete channel sequence $\left\{s_{1}, \ldots, s_{i-1}\right\}$, the sampling operations for the target choice $s_{i}$ within one Markov move is summarised as follows:

\section{Markov Mixing:}

1) Sample from the proposal distribution $Q^{\prime}\left(m_{t+1} \mid m_{t}\right)$ to obtain a candidate state $m^{*}$ for the Markov state $M_{t+1}$.

2) Calculate the acceptance ratio $\alpha^{\prime}\left(m^{*} \mid m_{t}\right)$

$$
\alpha^{\prime}\left(m^{*} \mid m_{t}\right)=\min \left\{1, \frac{\bar{P}\left(m^{*}\right) Q^{\prime}\left(m_{t} \mid m^{*}\right)}{\bar{P}\left(m_{t}\right) Q^{\prime}\left(m^{*} \mid m_{t}\right)}\right\} .
$$

3) With probability $\alpha^{\prime}\left(m^{*} \mid m_{t}\right)$ to accept $m^{*}$ by $M_{t+1}$, i.e. $M_{t+1}=m^{*}$, otherwise let $M_{t+1}=m_{t}$.

\section{Channel selection:}

4) If $m^{*}$ is accepted by $M_{t+1}$ and if $m^{*} \notin\left\{s_{1}, \ldots, s_{i-1}\right\}$, add $s_{i}=m^{*}$ into the channel sequence as $\mathcal{S}=\left[s_{1}, \ldots, s_{i}\right]$, otherwise try to obtain a valid $s_{i}$ at the next Markov move.

It should be noticed that similar to the original MH sampling, the Markov chain built by the RMH algorithm is also reversible by fulfilling the detailed balance condition [8]. Therefore, the Markov chain is valid as the Markovian property is reserved. To conclude, the details of the proposed RMH sampling algorithm for spectrum sensing is presented in Algorithm 1.

\section{B. Convergence Analysis and Performance Enhancement}

Besides the efficiency improvement, now we show that the proposed RMH sampling also achieves a better convergence 
performance than the MCMC sampling so that a better sampling accuracy can be achieved by the underlying Markov chain in the approximation of the target distribution $\bar{P}\left(s_{t}\right)$, which accounts for a better spectrum sensing performance. Intuitively, from the convergence shown in (4), a smaller convergence rate $\varrho$ means a better convergence performance to approximate the target distribution.

Theorem 1. Given the target distribution $\bar{P}(\cdot)$, the proposed RMH sampling achieves a better exponential convergence performance than $\mathrm{MH}$ by a smaller convergence rate

$$
\varrho_{\mathrm{RMH}}<\varrho_{\mathrm{MH}}
$$

Proof: First of all, according to (6) and (7), the transition probability of RMH sampling is derived as

$$
\begin{aligned}
& P_{\mathrm{RMH}}\left(m_{t}, m^{*}\right)=Q^{\prime}\left(m^{*} \mid m_{t}\right) \cdot \alpha^{\prime}\left(m^{*} \mid m_{t}\right) \\
& =\min \left\{Q^{\prime}\left(m^{*} \mid m_{t}\right), \frac{\bar{P}\left(m^{*}\right) Q^{\prime}\left(m_{t} \mid m^{*}\right)}{\bar{P}\left(m_{t}\right)}\right\} \\
& =\min \left\{\frac{Q\left(m^{*} \mid m_{t}\right)}{1-Q\left(m^{*}=m_{t} \mid m_{t}\right)}, \frac{\bar{P}\left(m^{*}\right) Q\left(m_{t} \mid m^{*}\right)}{\bar{P}\left(m_{t}\right)\left(1-Q\left(m_{t}=m^{*} \mid m^{*}\right)\right)}\right\} .
\end{aligned}
$$

Compared to the transition probability of the MH sampling

$$
\begin{aligned}
P_{\mathrm{MH}}\left(m_{t}, m^{*}\right) & =Q\left(m^{*} \mid m_{t}\right) \cdot \alpha\left(m^{*} \mid m_{t}\right) \\
& =\min \left\{Q\left(m^{*} \mid m_{t}\right), \frac{\bar{P}\left(m^{*}\right) Q\left(m_{t} \mid m^{*}\right)}{\bar{P}\left(m_{t}\right)}\right\},
\end{aligned}
$$

it is straightforward to see that

$$
P_{\mathrm{RMH}}\left(m_{t}, m^{*}\right)>P_{\mathrm{MH}}\left(m_{t}, m^{*}\right)
$$

for cases of $\mathcal{M}_{t}=m_{t} \neq \mathcal{M}_{t+1}=m^{*}$. More essentially, it means that each off-diagonal element in the transition matrix $\mathbf{P}_{\mathrm{RMH}}$ is always larger than that of $\mathbf{P}_{\mathrm{MH}}$. From literatures of MCMC, such a case is known as Peskun ordering written by

$$
P_{\mathrm{RMH}}\left(M_{t}, M_{t+1}\right) \succeq P_{\mathrm{MH}}\left(M_{t}, M_{t+1}\right) .
$$

Now, we invoke the following Lemma to reveal the relation between Peskun ordering and convergence rate.

Lemma 1 ([10]). Given reversible Markov chains $P$ and $G$ with stationary distribution $\pi$, if $P \succeq G$, then their convergence rates satisfy $\varrho_{P} \leq \varrho_{G}$.

Note that the definition of Peskun ordering $P\left(M_{t}, M_{t+1}\right) \succeq$ $G\left(M_{t}, M_{t+1}\right)$ given in [10] is based on the inequality $P\left(M_{t}, M_{t+1}\right) \geq G\left(M_{t}, M_{t+1}\right)$, where the equality $\varrho_{P}=\varrho_{G}$ holds only if $P\left(M_{t}, M_{t+1}\right)=G\left(M_{t}, M_{t+1}\right)$. Here, because the case of equality is not included, according to (13) and Lemma 1 , we can immediately obtain $\varrho_{\mathrm{RMH}}<\varrho_{\mathrm{MH}}$, completing the proof.

The insight behind Peskun ordering is that a Markov chain has smaller probability of remaining in the same position would explore the state space more efficiently, thus providing a better Markov mixing. This is straightforward to understand since if the Markov chain retains the same state over subsequent Markov moves, the autocorrelation along the exploration path will increase intensively, which naturally leads to a slow Markov mixing. Hence, according to Peskun ordering, the

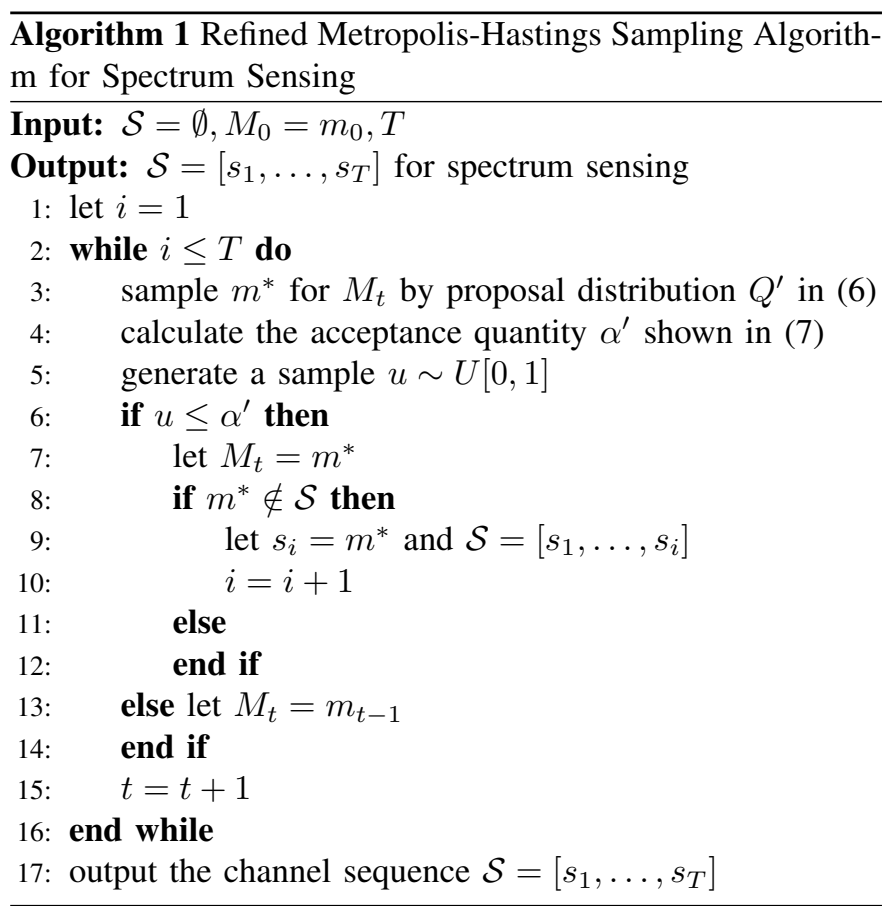

accuracy of spectrum sensing could be strengthened by the proposed RMH sampling algorithm as well.

\section{The Choice of the Proposal Distribution $Q$}

Next, the choice of the proposal distribution $Q(\cdot)$ is investigated, so as to determine the refined proposal distributions $Q^{\prime}$ in the proposed RMH sampling algorithm. In order to reduce the computational cost within each Markov move, the symmetric Gaussian proposal distribution $Q$ is considered, thus leading to an efficient Markov chain implementation. In particular, at each sampling stage, the proposal distribution $Q$ is designed as a conditional symmetric Gaussian distribution

$$
Q\left(m^{*} \mid m_{t}\right)=\frac{e^{-\frac{1}{2 \sigma^{2}}\left|m^{*}-m^{t}\right|^{2}}}{\sum_{m^{*}} e^{-\frac{1}{2 \sigma^{2}}\left|m^{*}-m^{t}\right|^{2}}}=Q\left(m_{t} \mid m^{*}\right),
$$

and the refined proposal distributions $Q^{\prime}\left(m^{*} \mid m_{t}\right)$ can be easily obtained based on (6). It is straightforward to verify that the refined proposal distributions $Q^{\prime}$ is also symmetric as well. In addition, the standard deviation $\sigma>0$ is flexible to set, which serves as temperature to adjust the Markov mixing [9].

By doing this, the sampling of $m^{*}$ heavily depends on the previous Markov state $m_{t}$. Meanwhile, due to the symmetric property, the calculation of the acceptance ratio $\alpha^{\prime}\left(m^{*} \mid m_{t}\right)$ is also greatly reduced by such an inhere elegance:

$$
\begin{aligned}
\alpha^{\prime}\left(m^{*} \mid m_{t}\right) & =\min \left\{1, \frac{\bar{P}\left(m^{*}\right) Q^{\prime}\left(m_{t} \mid m^{*}\right)}{\bar{P}\left(m_{t}\right) Q^{\prime}\left(m^{*} \mid m_{t}\right)}\right\} \\
& =\min \left\{1, \frac{f\left(m^{*}\right)}{f\left(m_{t}\right)}\right\} .
\end{aligned}
$$

Intuitively, $m^{*}$ is accepted by the Markov state $M^{t+1}$ if $f\left(m^{*}\right)>f\left(m_{t}\right)$, otherwise it would be accepted with probability $\frac{f\left(m^{*}\right)}{f\left(m_{t}\right)}$. Clearly, by fully making use of the symmetric Gaussian distribution, the sampling operations from 


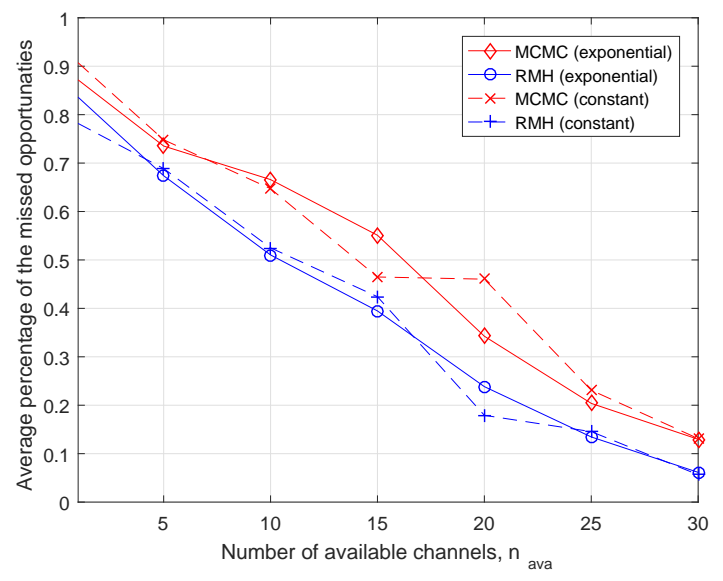

Fig. 1. Comparison of the average percentage of the missed opportunities over different traffic scenarios.

the Markov mixing could be significantly simplified for the complexity reduction, which further improves the sensing efficiency.

\section{Simulation Results}

To evaluate the effectiveness of the proposed RMH sampling algorithm for spectrum sensing, simulations are presented to illustrate the comparisons in terms of spectrum performance and efficiency. Specifically, we assume 40 channels in the licensed spectrum (i.e., $M=40$ ). The SINR is set to be $20 \mathrm{~dB}$ and the probability of false alarm is 0.01 . In Fig. 1, the average percentages of the missed spectrum opportunities under different traffic models (i.e., exponential packet arrival with an average arrival rate of 10 arrival/sec and constant packet arrival with an average arrival rate of 10 arrival/sec) are given for $n_{\max }=8$, where $n_{\max }$ denotes the maximum number of channels for fine sensing. Following the configurations in [6], the percentage of the missed spectrum opportunities $p_{m}$ is defined as

$$
p_{m}=1-\frac{n^{*}}{n_{\max }},
$$

where $n^{*}, n_{\text {req }}$ and $n_{\text {ava }}$ are the obtained number of available channels, the number of request available channels and the average number of available channels, respectively. As clearly can be seen, the proposed RMH sampling remains smaller probabilities of missing opportunities than the MCMC sampling in [6], implying a better sensing performance. On the other hand, the sensing efficiency is evaluated by the average sensing overhead in obtaining the available channels, where the sensing overhead $o$ is defined as

$$
o= \begin{cases}n_{\max }-n^{*} & \text { for } n^{*}=n_{\text {req }}, \\ n_{\max } & \text { for } n^{*} \neq n_{\text {req }} .\end{cases}
$$

From it, the average overhead in obtaining an available channel (i.e., $n^{*}=1$ ) with respect to the maximum number of fine sensing is shown in Fig. 2. As expected, the average sensing overhead of the proposed RMH sampling is noticeably lower than that of the MCMC sampling. Furthermore, as a complementary to show the efficiency improvement, Table I shows the

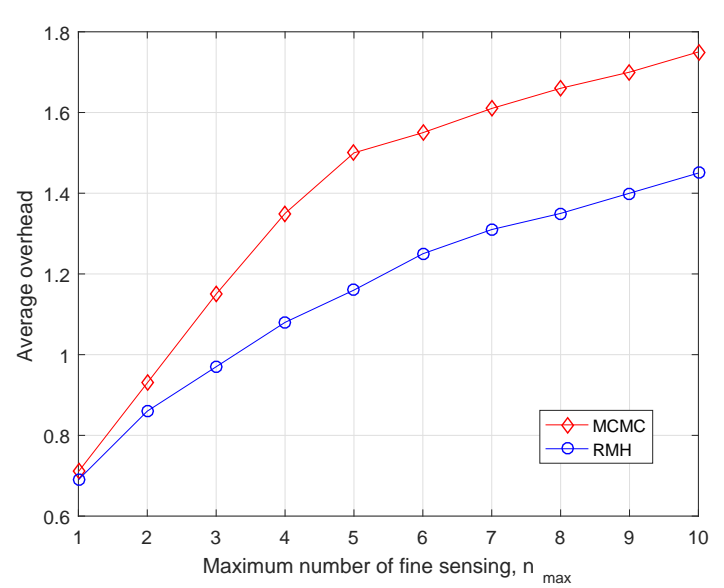

Fig. 2. Average overhead of obtaining an available channel with different maximum numbers of fine sensing.

TABLE I

AVERAGE PROBABILITIES OF BEING ACCEPTED BY THE CHANNEL SEQUENCE $\mathcal{S}$ DUE TO THE REQUIREMENT $m^{*} \notin\left\{s_{1}, \ldots, s_{i-1}\right\}$.

\begin{tabular}{|c||c||c||c||c||c||c|}
\hline & $i=1$ & $i=2$ & $i=3$ & $i=4$ & $i=5$ & $i=6$ \\
\hline MCMC & 1 & 0.71 & 0.50 & 0.41 & 0.35 & 0.28 \\
\hline RMH & 1 & 0.85 & 0.75 & 0.68 & 0.60 & 0.56 \\
\hline
\end{tabular}

acceptance rates due to the requirement $m^{*} \notin\left\{s_{1}, \ldots, s_{i-1}\right\}$ in both RMH and MCMC sampling. Intuitively, the proposed RMH sampling achieves a much more efficient sensing process than MCMC sampling due to a higher acceptance rate of the selection judgement in all cases of $i$.

\section{REFERENCES}

[1] A. Ali and W. Hamouda, "Advances on spectrum sensing for cognitive radio networks: Theory and applications," IEEE Communications Surveys Tutorials, vol. 19, no. 2, pp. 1277-1304, 2017.

[2] A. Patel and A. K. Jagannatham, "Non-antipodal signaling based robust detection for cooperative spectrum sensing in MIMO cognitive radio networks," IEEE Signal Processing Letters, vol. 20, no. 7, pp. 661-664, 2013.

[3] L. Arienzo and D. Tarchi, "Statistical modeling of spectrum sensing energy in multi-hop cognitive radio networks," IEEE Signal Processing Letters, vol. 22, no. 3, pp. 356-360, 2015.

[4] B. Sun, Q. Chen, X. Xu, Y. He, and J. Jiang, "Permuted filtered spectrum compressive sensing," IEEE Signal Processing Letters, vol. 20, no. 7, pp. 685-688, July 2013.

[5] Z. Li, B. Chang, S. Wang, A. Liu, F. Zeng, and G. Luo, "Dynamic compressive wide-band spectrum sensing based on channel energy reconstruction in cognitive internet of things," IEEE Transactions on Industrial Informatics, vol. 14, no. 6, pp. 2598-2607, 2018.

[6] X. Y. Wang, A. Wong, and P. Ho, "Spectrum sensing in cognitive radio using a Markov-chain Monte-Carlo scheme," IEEE Communications Letters, vol. 14, no. 9, pp. 830-832, 2010.

[7] C. Vladeanu, C. Nastase, and A. Martian, "Energy detection algorithm for spectrum sensing using three consecutive sensing events," IEEE Wireless Communications Letters, vol. 5, no. 3, pp. 284-287, 2016.

[8] D. A. Levin, Y. Peres, and E. L. Wilmer, Markov Chains and Mixing Time, American Mathematical Society, 2008.

[9] Z. Wang and C. Ling, "On the geometric ergodicity of MetropolisHastings algorithms for lattice Gaussian sampling," IEEE Transactions on Information Theory, vol. 64, no. 2, pp. 738-751, Feb. 2018.

[10] A. Mira, "Ordering and improving the performance of Monte Carlo Markov chains," Statistical Science, vol. 16, no. 4, pp. 340-350, 2001. 